\title{
The imbalance between proliferation and apoptosis contributes to degeneration of aortic valves and bioprostheses
}

\author{
Izabela Tuleta ${ }^{1}$, Abdel Karim Al Ghaddioui ${ }^{1}$, Gerhard Bauriedel ${ }^{2}$, Nicolas Wernert ${ }^{3}$, \\ Claus Jürgen Preusse ${ }^{4}$, Armin Welz ${ }^{4}$, Georg Nickenig ${ }^{1}$, Dirk Skowasch ${ }^{1}$ \\ ${ }^{1}$ Department of Internal Medicine II - Cardiology, Angiology and Pulmonology, \\ University of Bonn, Bonn, Germany \\ ${ }^{2}$ Department of Internal Medicine III - Invasive Cardiology and Cardiovascular Prevention, \\ Hospital of Schmalkalden, Schmalkalden, Germany \\ ${ }^{3}$ Department of Pathology, University of Bonn, Bonn, Germany \\ ${ }^{4}$ Department of Cardiac Surgery, University of Bonn, Bonn, Germany
}

\begin{abstract}
Background: The pathomechanisms underlying aortic valve degeneration are incompletely understood. Therefore, the aim of our work was to assess the quantitative changes of proliferation and apoptosis accompanied by cellular phenotype alternations and matrix secretion in aortic sclerotic and stenotic valves and degenerative bioprostheses, as well as to detect the expression pattern of the rapamycin receptor FKBP12 across these three valve types.
\end{abstract}

Methods: Mild-to-moderate sclerotic and stenotic valves and degenerative bioprostheses from 30 patients ( $n=10$ per group) were collected at autopsy or by surgery. Ki67+, FKBP12+, alpha-actin +, HSP47+ and TUNEL + apoptotic cells were analyzed by immunohistochemistry.

Results: The main finding was the reduced proliferation and increased apoptosis in stenotic valves (ST) compared to the sclerotic ones (SC) (proliferation: ST: $20.8 \pm 2.0 \%$ vs. SC: $30.1 \pm$ $\pm 2.2 \%$, apoptosis: ST: $40.7 \pm 5.0 \%$ vs. SC: $28.0 \pm 5.1 \%, p<0.05$, respectively). Analogical alternations were found in degenerative bioprostheses (BP) (proliferation: $4.8 \pm 2.3 \%$; apoptosis: $13.1 \pm 6.8 \%)$. Corresponding changes were observed in the valve cellularity (ST: $893 \pm$ $\pm 168, S C: 1034 \pm 284, B P: 385 \pm 179$ cells $/ \mathrm{mm}^{2}, p<0.05$, respectively). The FKBP12 signaling was reduced in diseased valves and bioprostheses (ST: $28.1 \pm 3.6 \%, S C: 42.2 \pm$ $\pm 3.8 \%$, BP: $5.8 \pm 1.9 \%, p<0.05$, respectively). Further, the augmented alpha-actin expression was observed as the degenerative process progressed (ST: $30.3 \pm 5.0 \%, S C: 22.6 \pm 2.7 \%, B P$ : $8.7 \pm 4.0 \%, p<0.05$, respectively), followed by the upregulation of HSP47 (ST: $22.6 \pm 2.8 \%$, SC: $15.4 \pm 2.1 \%, B P: 3.4 \pm 1.0 \%, p<0.05$, respectively) and consecutive matrix accumulation. Conclusions: The imbalance between proliferation and apoptosis with cellular phenotypical shift and subsequent matrix secretion may contribute to aortic valve stenosis and bioprosthesis degeneration. The identification of FKBP12 expression may implicate potential therapeutic strategies. (Cardiol J 2013; 20, 3: 268-275)

Key words: aortic valve degeneration, bioprosthesis, proliferation, apoptosis, rapamycin

Address for correspondence: Izabela Tuleta, MD, Department of Internal Medicine II - Cardiology, Angiology and Pulmonology, University of Bonn, Sigmund-Freud-Str. 25, 53105 Bonn, Germany, tel: 0049/228/287-16670, fax: 0049/228/287-16423, e-mail: Izabela.Tuleta@ukb.uni-bonn.de

Received: 30.09.2012

Accepted: 14.11.2012 


\section{Introduction}

Degenerative aortic valve stenosis is a very common cardiovascular disorder worldwide [1]. Despite significant advances in diagnosis and management, pathological processes underlying stenotic valve disease are still poorly understood [2]. Previous studies have identified several unique pathomechanisms that are believed to contribute to the progression of the aortic valve degeneration. Herein, the most important ones comprise apoptotic body formation with subsequent calcification, acquirement of the smooth muscle cell typical marker alpha-actin by valvular interstitial cells (VICs) and matrix disarrangement [3]. Aortic valve degeneration seems to be an active process involving both enhanced cell proliferation and apoptosis [4]. However, the extent and the time-dependent onset of these two processes have not been precisely explored yet.

Incomplete knowledge about the valve pathology impinges adequate treatment. Nowadays, valve replacement remains the only effective therapy [2]. Unfortunately, despite improvements in heart valve tissue engineering [5], a relatively fast bioprosthesis (BP) degeneration is a limitation to this method. That is why the understanding of the degeneration pattern of bioprostheses, particularly in comparison with the progress of native valve disease, could account for the development of new technologies supporting prolonged durability of valve substitutes. As an alternative to the invasive valve treatment, drug-based experimental therapy concepts such as the application of statins [6], blocking of Rho pathway [7] or TGF-beta1 signaling [8] have introduced a new promising therapeutic field. Since aortic valve lesions resemble arterial atherosclerotic plaques [9], potent antirestenotic drug - rapamycin - could represent another possible option to treat valve disease.

Against this background, the aim of our present study was to evaluate the histological characteristics involving the spatiotemporal pattern of proliferation, apoptosis and VIC activation by comparing mild-to-moderate, stenotic aortic valves and degenerative BP, as well as to detect FKBP12 receptors for rapamycin in these 3 valve types.

\section{Methods}

\section{Patients and valve specimens}

In the frame of a prospective study valve probes from a total of 30 patients were removed either during surgical valve replacement (10 native end-stage stenotic aortic valves, 10 degenera- tive porcine aortic bioprostheses) or at autopsy (10 mild-to-moderate aortic sclerotic valves). The diagnosis of aortic valve stenosis was made on the basis of a comprehensive anamnesis, physical examination, echocardiographic and invasive findings. Exclusion criteria comprised the presence of rheumatic disease, endocarditis, bicuspid valves and other coexisting valvular dysfunctions. The severity of valve calcification in stenotic aortic valves was graded semiquantitatively by echocardiography [10]. All of these valves showed moderate to severe calcification. Mild-to-moderate sclerotic aortic valves meeting the following macroscopic criteria: opaque leaflets with focal areas of mild thickening and increased stiffness, but no significant outflow obstruction and no relevant calcification [11] served as controls. There was no documented history of a clinically significant aortic valve stenosis among patients with mildto-moderate diseased valves. In order to compare the course of degeneration process in native valves to that in porcine bioprostheses, 7 Carpentier-Edwards and 3 Hancock/Hancock II devices with a mean duration of implant of $14 \pm 4$ years were explanted because of their degeneration-associated malfunction. The exact patients' characteristics are demonstrated in Table 1. Informed consent for the valve exploration was provided by each patient. The study was approved by the local ethics committee and conforms to the principles outlined in the Declaration of Helsinki.

\section{Immunohistochemistry}

The immunohistochemical examinations were conducted according to our previous protocols [12-14]. Specifically, valvular specimens were fixed in $4.5 \%$ buffered formaldehyde and embedded in paraffin. Serial sections $(4 \mu \mathrm{m})$ were taken vertically through the diseased cusp and sinus near the centre of the leaflet adjacent to calcified areas for immunohistochemical analysis. The specimens were not decalcified before sectioning to optimize antigen preservation. After preparation in xylol and alcohol, specimens underwent proteolysis with either 3\% citrate ( $\mathrm{pH} 6.0$ ) or target unmasking fluid (PanPath Inc., Amsterdam, the Netherlands). Non-specific antibody binding sites were blocked by fetal calf serum (FCS, dilution for FKBP12, alpha-actin and HSP47: 1:25) or rabbit serum (dilution for Ki67: 1:20) for $30 \mathrm{~min}$. Thereafter, the specific antibodies either of monoclonal (anti-Ki67: 1:2, clone MIB-1, Dako, Hamburg, Germany; anti-alpha-actin: 1:300, clone 1A4, Sigma, Diessenhofen, Germany; anti-HSP47: 1:1000, clone M16.10A1, Calbiochem, Schwalbach, Germany) 
Table 1. Characteristics of the patients' cohort.

\begin{tabular}{|c|c|c|c|c|}
\hline & $\operatorname{SC}(n=10)$ & ST $(n=10)$ & $\mathrm{BP}(\mathrm{n}=10)$ & $\mathbf{P}$ \\
\hline Age [years] & $61.4 \pm 12.5$ & $70.2 \pm 9.0$ & $65.5 \pm 8.7$ & 0.174 \\
\hline Male gender & $3(30 \%)$ & $8(80 \%)$ & $6(60 \%)$ & 0.076 \\
\hline Coronary artery disease & $3(30 \%)$ & $7(70 \%)$ & $4(40 \%)$ & 0.175 \\
\hline Peripheral artery occlusive disease & $0(0 \%)$ & $1(10 \%)$ & $1(10 \%)$ & 0.585 \\
\hline \multicolumn{5}{|l|}{ Cardiovascular risk factors } \\
\hline Arterial hypertension & $3(30 \%)$ & $8(80 \%)$ & $7(70 \%)$ & 0.054 \\
\hline Diabetes mellitus & $2(20 \%)$ & $5(50 \%)$ & $1(10 \%)$ & 0.109 \\
\hline Hyperlipidemia & $1(10 \%)$ & $5(50 \%)$ & $5(50 \%)$ & 0.101 \\
\hline Smoker & $1(10 \%)$ & $4(40 \%)$ & $3(30 \%)$ & 0.303 \\
\hline Obesity & $1(10 \%)$ & $1(10 \%)$ & $4(40 \%)$ & 0.153 \\
\hline Familial disposition & $0(0 \%)$ & $3(30 \%)$ & $1(10 \%)$ & 0.133 \\
\hline \multicolumn{5}{|l|}{ Medication } \\
\hline Aspirin & $4(40 \%)$ & $7(70 \%)$ & $6(60 \%)$ & 0.387 \\
\hline ACE inhibitor & $3(30 \%)$ & $6(60 \%)$ & $7(70 \%)$ & 0.175 \\
\hline Beta-blocker & $4(40 \%)$ & $8(80 \%)$ & $6(60 \%)$ & 0.189 \\
\hline Statin & $3(30 \%)$ & $7(70 \%)$ & $4(40 \%)$ & 0.175 \\
\hline
\end{tabular}

Values are presented as a mean $\pm \mathrm{SD}$. Values in brackets conform to percentages; SC — mild-to-moderate aortic sclerosis; ST — end-stage aortic stenosis; BP — degenerative aortic bioprosthesis; ACE — angiotensin-converting enzyme; $\mathrm{P}$ - probability value

or polyclonal (anti-FKBP12: 1:500, Biomol, Hamburg, Germany) origin were used. For polyclonal antibodies, AffiniPure mouse anti-rabbit IgG (Dianova) was additionally applied for $30 \mathrm{~min}$ at room temperature following primary antibody incubation. The visualization of antibody-protein complexes was performed by means of the APAAP technique (Alkaline Phosphatase Anti-Alkaline Phosphatase; Boehringer, Mannheim, Germany) with Fast Red (4-Chloro-2-Methylbenzendiazonium/3-Hydroxy-2-Naphthoesäure2,4-Dimethylanilid Phospat; Sigma, Diessenhofen, Germany) as a chromogenic substrate. The nuclei were counterstained with hematoxylin (Merck, Darmstadt, Germany). Negative controls consisted in omitting of the specific antibodies.

In situ cell death detection (TUNEL-Assay:

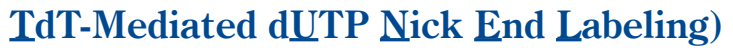

The immunohistochemical identification of apoptotic cells was performed in terms of labeling of DNA strand breaks with modified nucleotides. In detail, following the application of proteinase $\mathrm{K}$ $\left(1: 1000,30 \mathrm{~min}\right.$ at $\left.37^{\circ} \mathrm{C}\right)$ the unspecific antibody sites were blocked with FCS $(1: 25,30 \mathrm{~min}$ at $20^{\circ} \mathrm{C}$ ). Subsequently, the terminal deoxynucleotidyl transferase (TdT, Boehringer, Mannheim, Germany) was used as a catalyst of polymerization of fluorescein labeled nucleotides (Boehringer, Mannheim, Germany) to free 3'-OH DNA ends. Then, the fluorescein labeled nucleotides were detected by anti-fluorescein antibody Fab fragments from sheep (Boehringer, Mannheim, Germany) conjugated with alcaline phosphatase $\left(30 \mathrm{~min}, 37^{\circ} \mathrm{C}\right)$. Eventually, a colour reaction was conducted with Fast Red (Sigma, Diessenhofen, Germany). Nuclei were stained with hematoxylin. In each experiment 2 negative controls were carried out with labeled nucleotides without incubation with TdT.

\section{Histology}

Photomicrographs were obtained by an Optiphot-2 microscope (Nikon, Düsseldorf, Germany) and a downstream KP-C 553 CCD video camera (Hitachi, Rodgau, Germany) [11-13]. A computer-assisted morphometric system (VFG 1 graphic card/VIBAM 0.0 software) was used to calculate cell density and expression of the determined markers. Five randomly selected areas $\left(0.04 \mathrm{~mm}^{2}\right.$ each) were explored in each valve layer: ventricularis, spongiosa, fibrosa. The percentage of immunolabeled cells was assessed as a number of stained cells per total cell number within each layer. Morphometric analysis was performed by 2 independent examiners.

In order to distinguish valve layers from each other Azan trichome staining for collagen fibres and van Gieson elastica staining for elastic fibres were conducted. 


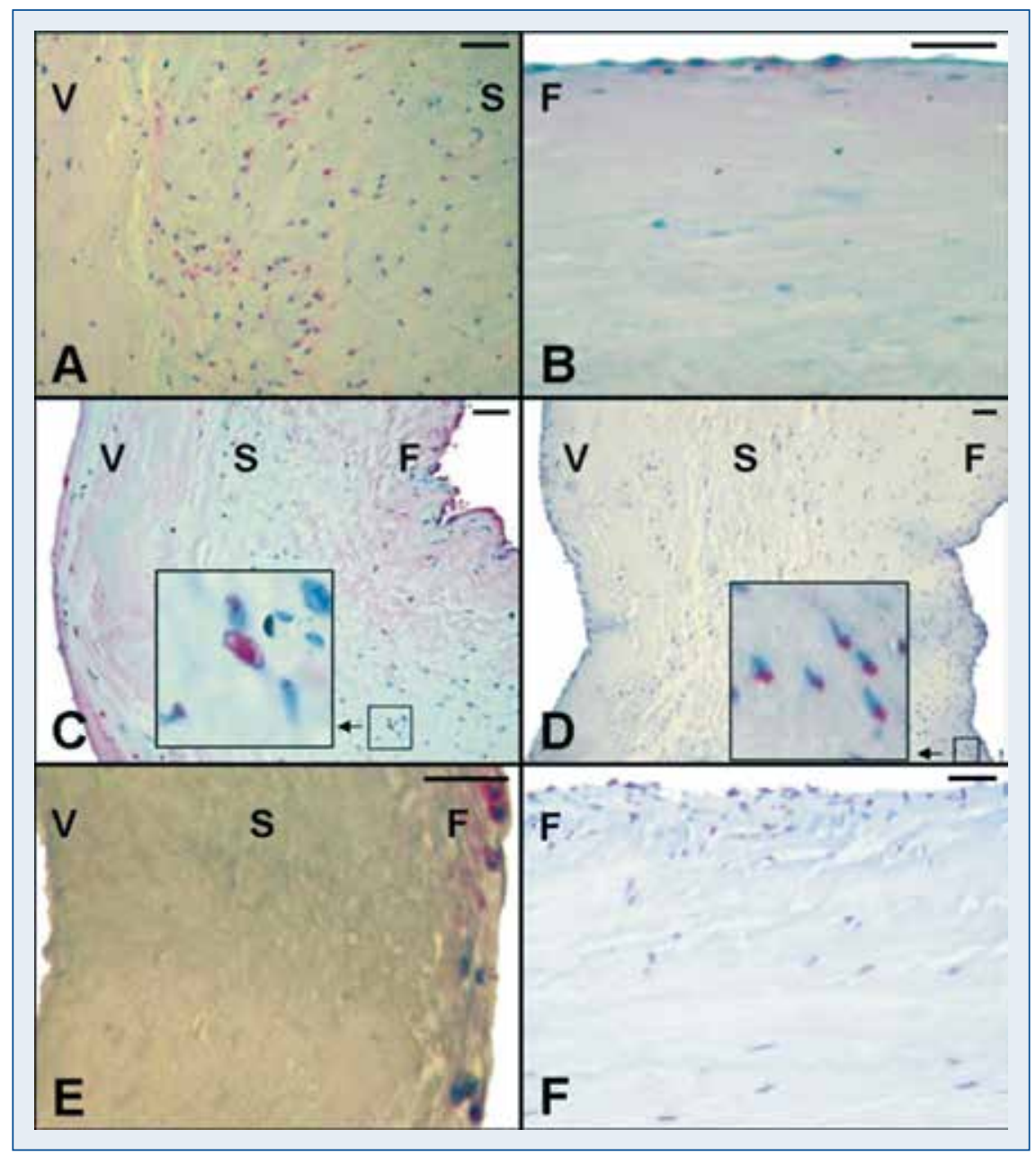

Figure 1. Photomicrographs demonstrating a decreasing number of Ki67+ proliferating cells (A. Sclerotic valve; B. Stenotic valve) and an increasing TUNEL+ apoptotic activity (C. Sclerotic valve; D. Stenotic valve) in a time course of the degeneration process of a native aortic valve. Analogically, the prevalence of apoptosis (F) over proliferation (E) in a porcine degenerative bioprosthesis; $\mathrm{F}$ - fibrosa; $\mathrm{S}$ - spongiosa; $\mathrm{V}$ - ventricularis; $\mathrm{Bar}=30 \mu \mathrm{m}$.

\section{Statistical analysis}

Statistical analysis was performed by SPSS Inc. (version 19.0). All parameters were presented as a mean value \pm standard derivation. In order to assess possible differences in marker expression levels between 3 groups of valves, the 1-way ANOVA test with the post-hoc Tukey-B analysis was used. Categorical variables were compared across the groups by means of the $\chi^{2}$ test. $\mathrm{P}$ value of under 0.05 was considered to be statistically significant. Two-tailed bivariate correlations were determined by the Pearson's coefficient.

\section{Results}

The main finding of our study was the decreasing proliferation rate and the increasing apoptotic index in the time course of the degeneration process of the native human aortic valves (Figs. $1 \mathrm{~A}-\mathrm{D}$ ). About one third of all VICs were proliferating in the mild-to-moderate sclerotic valves, as indicated by Ki67+ cells (SC: $30.1 \pm 2.2 \%$, Figs. $1 \mathrm{~A}, 2$ ). The number of mitotic cells reduced in stenotic valves (ST: $20.8 \pm 2.0 \%$, Figs. $1 \mathrm{~B}, 2$ ). On the contrary, apoptotic activity, expressed by a percentage of TUNEL + cells, was detected in $28.0 \pm 5.1 \%$ of VICs in sclerotic valves and increased as degeneration process proceeded (ST: $40.7 \pm 5.0 \%$, Figs. $1 \mathrm{C}$, $\mathrm{D}, 2)$. In consequence, the apoptosis/proliferation ratio amounted to about 1 in only mild-to-moderate altered valves, whereas it increased to approximately 2 at the end stage of valve disease. Similarly, the apoptosis outnumbered the proliferation rate in bioprostheses by about 2 times (BP: apoptosis: $13.1 \pm 6.8 \%$, proliferation: $4.8 \pm 2.3 \%$, Figs. $1 \mathrm{E}, \mathrm{F}, 2$ ) The above differences in proliferation and apoptosis 


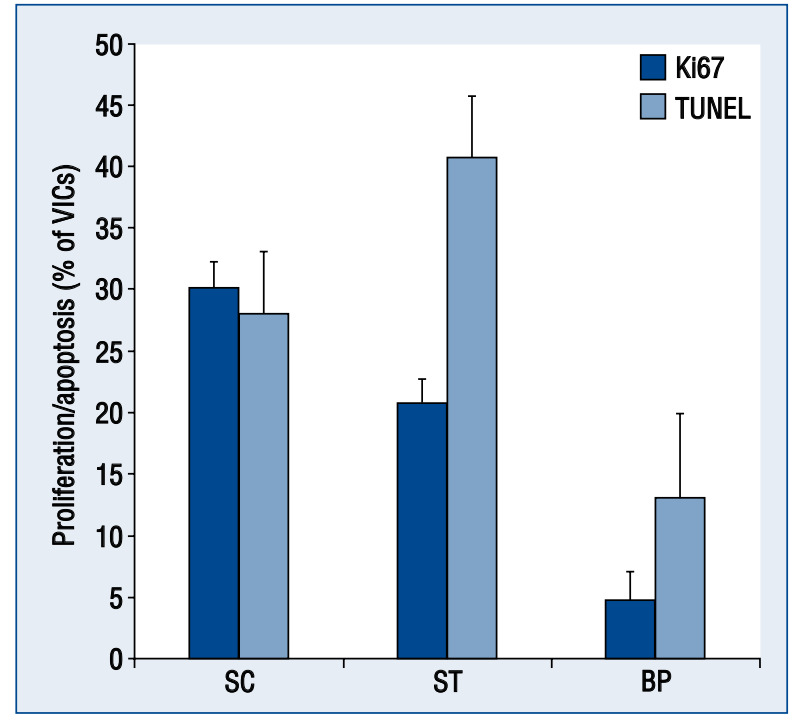

Figure 2. A graphic chart representing a percentual relationship between proliferation (Ki67) and apoptosis (TUNEL) in valvular interstitial cells (VICs) of sclerotic (SC) and stenotic valves (ST) as well as bioprostheses (BP).

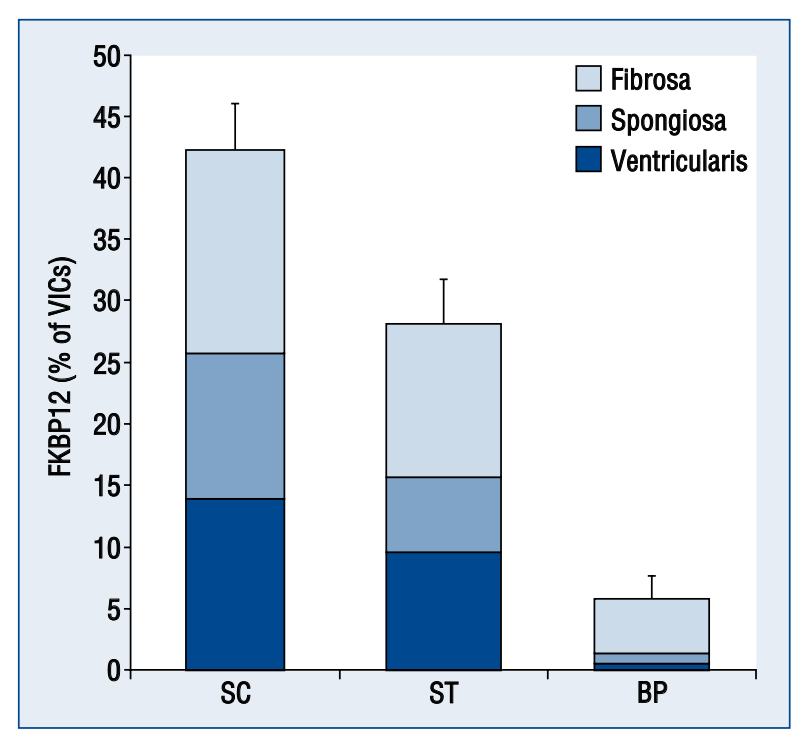

Figure 4. Percentual fraction of FKBP12 positive cells among valvular interstitial cells (VICs) in 3 valve layers: ventricularis, spongiosa, fibrosa across 3 types of valves; SC - sclerotic valves; ST — stenotic valves; $\mathrm{BP}$ - bioprostheses.

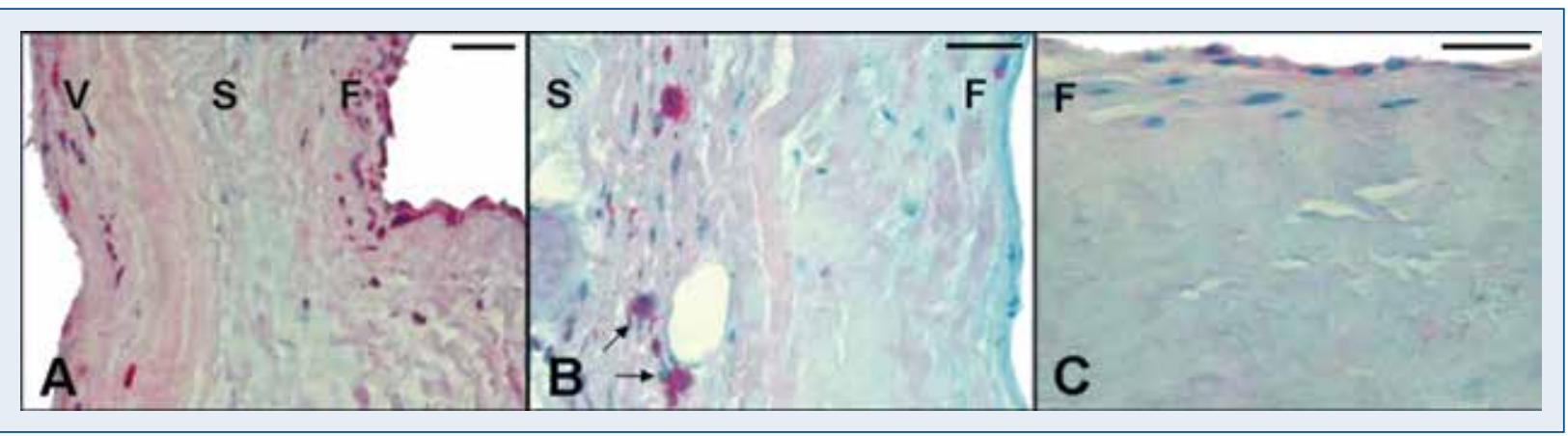

Figure 3. Declining FKBP12 signal density in a native stenotic aortic valve (B) compared to a sclerotic one (A). C. Distinct FKBP12 immunolabeling in a bioprosthesis. Remarkably, fibrosa is a predilection site for FKBP12 signaling in all 3 valve types. Arrows indicate FKBP12+ cells adjacent to a microvessel in a border zone between fibrosa and spongiosa; F - fibrosa; S - spongiosa; V - ventricularis; Bar $=30 \mu \mathrm{m}$.

were significant across the 3 valve types ( $p<0.05$, respectively). A comparison of the corresponding changes in proliferation and apoptosis revealed a strong positive correlation between these 2 processes in sclerotic valves $(r=0.7, p=0.02)$ and a strong negative correlation in the stenotic ones $(\mathrm{r}=-0.7, \mathrm{p}=0.02)$. The described interplay between proliferation and apoptosis resulted in changes in the valve cellularity (SC: $1034 \pm 284$ cells $/ \mathrm{mm}^{2}$, ST: $893 \pm 168$ cells $/ \mathrm{mm}^{2}$, BP: $385 \pm$ \pm 179 cells $\left./ \mathrm{mm}^{2}, \mathrm{p}<0.05\right)$.

Parallel to the enhanced cell turnover, we observed the upregulation of the signaling for
FKBP12, the receptor for rapamycin, in sclerotic valves (SC: $42.2 \pm 3.8 \%$ ) which declined significantly in the stenotic ones (ST: $28.1 \pm 3.6 \%$, Figs. 3A, B, 4). In the bioprostheses the FKBP12 immunolabeling was detected in $5.8 \pm 1.9 \%$ of cells $(\mathrm{p}<0.05$ between 3 valve types, Figs. 3C, 4$)$. Interestingly, the FKBP12 signals predominated in the fibrosa of both valve types and at the aortic site of the bioprostheses $(\mathrm{p}<0.05$ compared to the remaining layers, respectively, Figs. 3, 4).

Moreover, we identified the relevant part of VICs to be positive for alpha-actin marker in all 3 types of explored valves (SC: $22.6 \pm 2.7 \%$, ST: 


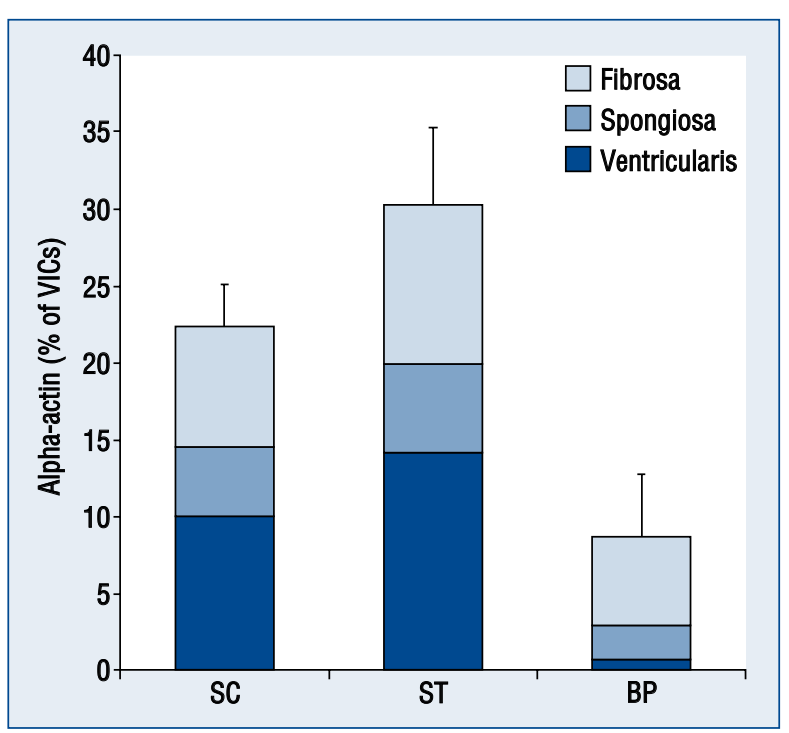

Figure 5. Percentual fraction of alpha-actin positive cells among valvular interstitial cells (VICs) in 3 valve layers: ventricularis, spongiosa, fibrosa across 3 types of; $\mathrm{SC}$ - sclerotic valves; ST — stenotic valves; $\mathrm{BP}$ - bioprostheses.

$30.3 \pm 5.0 \%$, BP: $8.7 \pm 4.0 \%, \mathrm{p}<0.05)$. These myofibroblast-like alpha-actin + cells were mostly located in the ventricularis of the sclerotic and stenotic valves (alpha-actin ratio in ventricularis: spongiosa: fibrosa for SC: 2.2: 1.0: 1.8, $\mathrm{p}<0.001$; for ST: 2.5: 1.0: 1.8, $\mathrm{p}<0.001$ ), whereas in the bioprostheses the layers close to aorta were the predilection sites for this cell type (BP: 1.0: 3.4: 9.1, respectively, $p<0.001$, Figs. 5, 6).

Along with the increase in the alpha-actin expression, the upregulation of a collagen-specific molecular chaperon HSP47, particularly in the fibrosa of the valves (SC: $15.4 \pm 2.1 \%$, ST: $22.6 \pm$ $\pm 2.8 \%$, BP: $3.4 \pm 1.0 \%, \mathrm{p}<0.05)$ with consecutive matrix accumulation, was detected.

\section{Discussion}

Our present study has provided new insights into the degenerative process of native human aortic valves with emphasis on the cell turnover in time course, potential differences in the disease progression between native valves and bioprostheses and possible therapeutic strategies. Particularly, we have demonstrated that in the initial stage of the degenerative process the proliferation rate of about $30 \%$ of all interstitial valve cells was well balanced by apoptosis. This indicates that, in comparison with healthy aortic valves which are characterized by very low proliferation and apoptosis indexes [15], the cell turnover rate increases rapidly in the early phase of the valve disease, similarly as during fetal development [15]. Likewise, enhanced cell proliferation was observed in pediatric and adult diseased aortic valves [4].

Our data show that with time in stenotic aortic valves proliferation decreased and apoptosis increased with apoptosis/proliferation ratio of approximately 2 , analogically to that found in bioprostheses. In mild-to-moderate degenerative valves the correlation between proliferation and apoptosis was significantly positive. In contrast, it was strongly negative in the stenotic valves. It seems that at the beginning of the degenerative process the proliferation and apoptosis as 2 counteracting processes balance each other. However, it is not known whether the cell apoptosis occurs at first as a reaction to the undefined trauma followed by the attempt of rebalance due to the activation of proliferation or on the contrary, apoptosis is secondary to the cell proliferation which takes

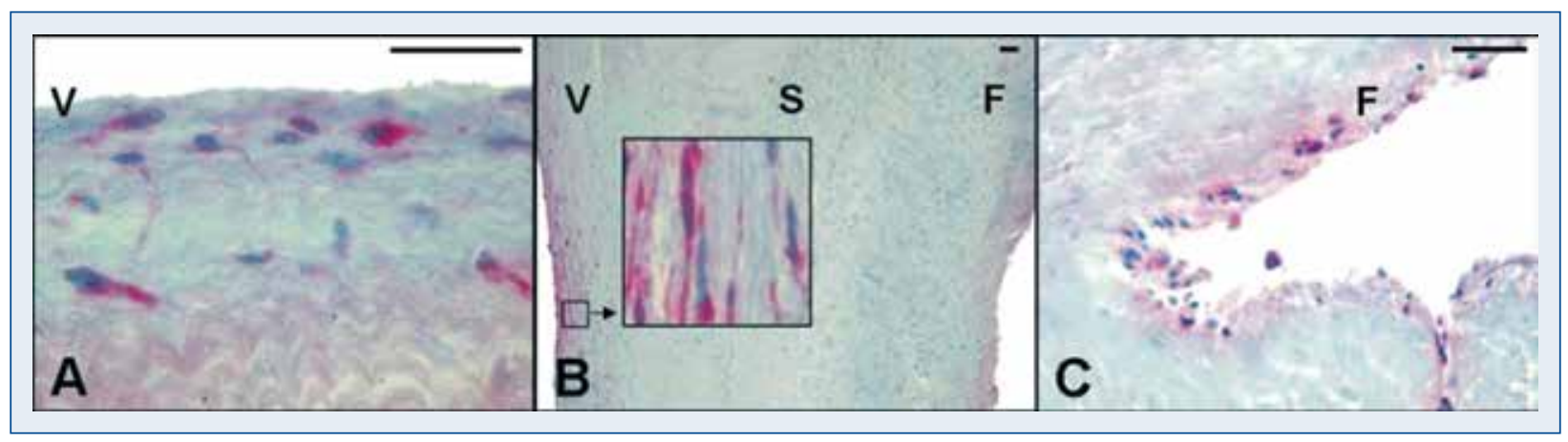

Figure 6. Predominance of alpha-actin signals in a ventricularis of a sclerotic valve (A) and of a stenotic valve (B). In contrast, alpha-actin + cells are almost exclusively found in a fibrosa of a bioprosthesis (C); $\mathrm{F}$ - fibrosa; $\mathrm{S}-\mathrm{spon-}$ giosa; V - ventricularis; Bar $=30 \mu \mathrm{m}$. 
place in order to reproduce cell population capable of renewing of the damaged extracellular matrix. In stenotic aortic valves the negative correlation between apoptosis and proliferation points to the dysregulation in cell turnover and subsequently to the irreversible disturbance of a valve homeostasis. Increased apoptosis seems to be associated with augmented calcification [3]. One may assume that whereas the mild-to-moderate stage of degenerative valve disease with in fact pathologically augmented cell activation but still sustained cell balance is potentially reversible, the stenotic valve with the predominance of apoptosis represents a "point of no return" in the time course of the degeneration process. The diagnosis based on evaluation of such indicator of valve disease progression could be of a great importance for identification of patients with not yet advanced disease who could profit from early treatment other than surgical valve replacement. Likewise, Wirrig et al. [4] proposed the expression of osteogenic differentiation molecules to be a crucial marker indicating a transition from a non-calcified state to the late stage of a calcified aortic valve disease.

Another finding of our study was the detection of FKBP12 signaling in diseased valves, which could implicate potential therapeutic strategies. The exact role of FKBP12 in the pathophysiology of cardiovascular system is not known [16]. As a receptor for rapamycin FKBP12 regulates various processes connected with cell growth, proliferation and protein synthesis [17]. In analogy to the prevention of vessel restenosis, the use of rapamycin could be of a therapeutic relevance. The rapamycin-associated antiproliferative actions [18] might cease the exaggerated reaction of mutual intensification of proliferation and apoptosis. Keeping in mind the spatiotemporal pattern of FKBP12 receptor and the apoptosis/proliferation ratios, rapamycin application should be restricted to the early stage of degenerative valve process at the fibrosa site. Moreover, the anti-inflammatory properties of rapamycin could attenuate the inflammation contributing to the valve disease [14].

In our study, besides the unbalance between proliferation and apoptosis, the upregulation of alpha-actin + cells was related to the degeneration process. Interestingly, the majority of alpha-actin signals were found in the ventricularis of the diseased native valves. In contrast, alpha-actin immunolabeling was almost only detected in the fibrosa of bioprostheses. This fact suggests different origins of alpha-actin + cells and/or their precursors in the diseased native valves and bioprostheses. Specifically, alpha-actin + cells could migrate from neighboring cardiac tissue into ventricularis of native valves through the reactivation of embryonic development pathways consisting in population of valve leaflets with endocardial cells that have undergone an epithelial-to-mesenchymal transformation [19]. In contrast, the localization of alpha-actin markers in the fibrosa of bioprostheses indicates extra-cardiac source of these cells such as peripheral blood $[12,14]$ or aorta. It is also possible that different predilection sites for alpha-actin+ cells in impaired native valves and bioprotheses are a consequence of a valve type specific distribution of factors promoting phenotypical shift from quiescent fibroblast-like cells which are typical for healthy valves [20] into myofibroblast-like cells. Either way, different parts of valves seem to accomplish an unequal contribution to the degeneration process dependent on the native or bioprosthetic valve type.

Our work is limited to the investigations of the valve changes at certain time points without assessment of the progression from sclerotic to the end-stage stenotic valve disease. Moreover, the number of specimens examined was relatively small. Eventually, the study design comprised only morphological and immunohistochemical analysis. Nevertheless, the present work suggests some interesting valve degeneration pathomechanisms which should be further evaluated in larger studies with emphasis on the functional aspects under the application of molecular biological methods.

\section{Conclusions}

Our study shows an imbalance between proliferation and apoptosis accompanied by the alpha-actin upregulation and matrix secretion in degenerative valves and bioprostheses. Identification and determination of FKBP12 spatiotemporal distribution implicate its role in the valve degeneration process and provide a platform for new therapeutic strategies. The differences in the alpha-actin expression suggest different degeneration patterns in native valves and bioprostheses.

\section{Acknowledgements}

The authors gratefully acknowledge the statistical help of Dr R. Fimmers, Institute of Medical Biometry, Informatics and Epidemiology, University of Bonn.

Conflict of interest: none declared 


\section{References}

1. Iung B, Vahanian A. Valvular heart disease in elderly people. Lancet, 2006; 368: 969-971.

2. Bonow RO, Carabello BA, Kanu C et al. ACC/AHA 2006 guidelines for the management of patients with valvular heart disease: A report of the American College of Cardiology/American Heart Association Task Force on Practice Guidelines (writing committee to revise the 1998 Guidelines for the Management of Patients With Valvular Heart Disease): Developed in collaboration with the Society of Cardiovascular Anesthesiologists: endorsed by the Society for Cardiovascular Angiography and Interventions and the Society of Thoracic Surgeons. Circulation, 2006; 114: e84-e231.

3. Liu AC, Joag VR, Gotlieb AI. The emerging role of valve interstitial cell phenotypes in regulating heart valve pathobiology. Am J Pathol, 2007; 171: 1407-1418.

4. Wirrig EE, Hinton RB, Yutzey KE. Differential expression of cartilage and bone-related proteins in pediatric and adult diseased aortic valves. J Mol Cell Cardiol, 2011; 50: 561-569.

5. Gandaglia A, Bagno A, Naso F, Spina M, Gerosa G. Cells, scaffolds and bioreactors for tissue-engineered heart valves: A journey from basic concepts to contemporary developmental innovations. Eur J Cardiothorac Surg, 2011; 39: 523-531.

6. Osman L, Yacoub MH, Latif N, Amrani M, Chester AH. Role of human valve interstitial cells in valve calcification and their response to atorvastatin. Circulation, 2006; 114: I547-I552.

7. Gu X, Masters KS. Role of the Rho pathway in regulating valvular interstitial cell phenotype and nodule formation. Am J Physiol Heart Circ Physiol, 2011; 300: H448-H458.

8. Cushing MC, Mariner PD, Liao JT, Sims EA, Anseth KS. Fibroblast growth factor represses Smad-mediated myofibroblast activation in aortic valvular interstitial cells. FASEB J, 2008; 22: 1769-1777.

9. Breyne J, Juthier F, Corseaux D et al. Atherosclerotic-like process in aortic stenosis: Activation of the tissue factor-throm- bin pathway and potential role through osteopontin alteration. Atherosclerosis, 2010; 213: 369-376.

10. Baumgartner $\mathrm{H}$, Hung J, Bermejo J et al. EAE/ASE recommendations for clinical practice. Eur J Echocardiogr, 2009; 10: 1-25.

11. Kuusisto J, Räsänen K, Sarkioja T, Alarakkola E, Kosma VM. Atherosclerosis-like lesions of the aortic valve are common in adults of all ages: A necropsy study. Heart, 2005; 91: 576-582.

12. Skowasch D, Schrempf S, Wernert $\mathrm{N}$ et al. Cells of primarily extravalvular origin in degenerative aortic valves and bioprostheses. Eur Heart J, 2005; 26: 2576-2580.

13. Tuleta I, Skowasch D, Peuster M, Nickenig G, Bauriedel G. Cells of primarily extravascular origin in neointima formation following stent implantation: Coordinated expression of endothelial progenitor, dendritic and neural crest-derived cells. Cardiology, 2008; 110: 199-205.

14. Skowasch D, Tuleta I, Steinmetz M et al. Pathogen burden in degenerative aortic valves is associated with inflammatory and immune reactions. J Heart Valve Dis, 2009; 18: 411-417.

15. Aikawa $\mathrm{E}$, Whittaker $\mathrm{P}$, Farber $\mathrm{M}$ et al. Human semilunar cardiac valve remodeling by activated cells from fetus to adult: Implications for postnatal adaptation, pathology, and tissue engineering. Circulation, 2006; 113: 1344-1352.

16. Wang T, Donahoe PK. The immunophilin FKBP12: A molecular guardian of the TGF-beta family type I receptors. Front Biosci, 2004; 9: 619-631.

17. Drenan RM, Liu X, Bertram PG, Zheng XF. FKBP12-rapamycinassociated protein or mammalian target of rapamycin (FRAP/ /mTOR) localization in the endoplasmic reticulum and the Golgi apparatus. J Biol Chem, 2004; 279: 772-778.

18. Sehgal SN. Sirolimus: Its discovery, biological properties, and mechanism of action. Transplant Proc, 2003; 35: 7S-14S.

19. Eisenberg LM, Markwald RR. Molecular regulation of atrioventricular valvuloseptal morphogenesis. Circ Res, 1995; 77: $1-6$.

20. Merryman WD. Mechano-potential etiologies of aortic valve disease. J Biomech, 2010; 43: 87-92. 